\title{
High energy $\mathrm{X}-\gamma$ ray spectrometer on the Chandrayaan-1 mission to the Moon
}

\author{
J N Goswami ${ }^{1, *}$, D Banerjee ${ }^{1}, \mathrm{~N}$ Bhandari $^{1}$, M Shanmugam $^{1}$, Y B Acharya ${ }^{1}$, \\ D V Subhedar ${ }^{1}$, M R Sharma ${ }^{2}$, C N Umapathy ${ }^{2}$, P Sreekumar ${ }^{2}$, M Sudhakar ${ }^{2}$, \\ L ABRAHAM ${ }^{2}$ and P C AGRAWAL \\ ${ }^{1}$ Physical Research Laboratory, Ahmedabad 380 009, India. \\ ${ }^{2}$ Space Astronomy and Instrumentation Division, ISRO Satellite Center, Bangalore 560 017, India. \\ ${ }^{3}$ Tata Institute of Fundamental Research, Mumbai 400 005, India. \\ *e-mail: goswami@prl.ernet.in
}

The Chandrayaan-1 mission to the Moon scheduled for launch in late 2007 will include a high energy X-ray spectrometer (HEX) for detection of naturally occurring emissions from the lunar surface due to radioactive decay of the ${ }^{238} \mathrm{U}$ and ${ }^{232} \mathrm{Th}$ series nuclides in the energy region $20-250 \mathrm{keV}$. The primary science objective is to study the transport of volatiles on the lunar surface by detection of the $46.5 \mathrm{keV}$ line from radioactive ${ }^{210} \mathrm{~Pb}$, a decay product of the gaseous ${ }^{222} \mathrm{Rn}$, both of which are members of the ${ }^{238} \mathrm{U}$ decay series. Mapping of $U$ and $T h$ concentration over the lunar surface, particularly in the polar and U-Th rich regions will also be attempted through detection of prominent lines from the $U$ and Th decay series in the above energy range. The low signal strengths of these emissions require a detector with high sensitivity and good energy resolution. Pixelated CadmiumZinc-Telluride (CZT) array detectors having these characteristics will be used in this experiment. Here we describe the science considerations that led to this experiment, anticipated flux and background (lunar continuum), the choice of detectors, the proposed payload configuration and plans for its realization.

\section{Introduction}

The Indian Space Research Organisation (ISRO) has started a new initiative to launch dedicated scientific satellites earmarked for planetary exploration and plans to launch the Chandrayaan-1 mission to Moon in late 2007. The basic objective of this mission is photo-selenological and chemical mapping of the Moon with better spatial and spectral resolution. Consistent with this scientific objective, several baseline Indian payloads are included in this mission. The high-energy Xray $(20-250 \mathrm{keV})$ spectrometer (HEX) is one of the baseline payloads intended primarily for the study of volatile transport on the Moon, and to infer $\mathrm{U}$ and Th concentrations in the polar and U-Th-rich regions on the Moon with low spatial resolution.
Chemical composition of various solar system bodies such as planets, satellites and asteroids provides important clues towards understanding their origin and evolution (Haines et al 1976). High energy $(>500 \mathrm{keV}) \gamma$-ray spectroscopy is a prime tool for remote sensing studies of chemical composition of planetary surfaces and has been utilized to study surface composition of the Moon, Mars and Asteroids at various spatial resolutions. In the case of the Moon, the Apollo and Luna missions mapped about $20 \%$ of the equatorial lunar surface for concentrations of $\mathrm{K}, \mathrm{U}, \mathrm{Th}, \mathrm{Fe}, \mathrm{Mg}$, Ti and several other elements (Arnold et al 1977). More recently, the Lunar Prospector mission obtained a global map of $\mathrm{Fe}, \mathrm{Th}, \mathrm{U}, \mathrm{K}$ and a few other elements (O, Si, Al, Ca, Mg, Ti) with a footprint of 150 by $150 \mathrm{~km}$ (Lawrence et al 1998). Analysis

Keywords. Chemical composition; volatile transport; high energy X-rays; Chandrayaan-1; thorium distribution on Moon. 
of Lunar Prospector data has also demonstrated a correlation between K, U and Th. While the Apollo mission experiments used NaI scintillator as the detector, a Bismuth Germanate (BGO) scintillation detector having better energy resolution was used in the Lunar Prospector mission. The recent Mars Odyssey mission had a $\gamma$-ray spectrometer that used a large germanium crystal cooled passively to $\sim 85 \mathrm{~K}$ (Boynton et al 2002). At present, detection of characteristic $\gamma$-ray lines from planetary surfaces in the low energy $(<500 \mathrm{keV})$ region has not been attempted because of their low signal strength and the anticipated high detector and planetary continuum background. With the development of new solid state array detectors, it is now possible to explore this energy region, and the High Energy $\mathrm{X}-\gamma$ ray (HEX) experiment to be flown in the Chandrayaan-1 mission is designed to study the emission of low energy $(20-250 \mathrm{keV})$ natural $\gamma$ rays from the lunar surface. This will represent the first attempt to study low energy $\gamma$-ray emission from a planetary surface.

\section{Science objectives of the HEX experiment}

The existence of permanently shadowed areas in the polar regions of the Moon led to the suggestion that these areas can act as cold traps for water molecules and other volatiles that will be transported from the hot sunlit side of the Moon to these colder areas. Further, it was suggested that there could be substantial reservoir of water ice in the polar regions of the Moon (Watson et al 1961; Arnold 1979). A claim for the existence of water on lunar poles was made on the basis of the bistatic radar experiment conducted during Clementine mission (Nozette et al 1996). However, subsequent earth-based radar observations have questioned the validity of this claim (Stacey et al 1997). Neutron spectroscopy studies carried out by the Lunar Prospector mission suggested the presence of hydrogen in the polar region, strengthening the case of presence of water ice in these areas (Feldman et al 1998). The presence of water in the lunar polar region, if confirmed, is extremely important for the utilization of Moon as a base for scientific and technological exploration and long-term human presence on the Moon. The HEX experiment on Chandrayaan- 1 is designed to investigate the phenomenon of transport of volatiles on the lunar surface through the detection of the $46.5 \mathrm{keV} \gamma$-ray line from radioactive ${ }^{210} \mathrm{~Pb}$ (Bhandari et al 2003), a decay product of the volatile ${ }^{222} \mathrm{Rn}$ having a boiling point of $-61.8^{\circ} \mathrm{C}$ and a half-life of $\sim 3.8$ days, both belonging to the ${ }^{238} \mathrm{U}$ series. The half-life of ${ }^{222} \mathrm{Rn}$ is long enough to effectively transport most of the radon produced in the upper meter of the lunar regolith to the surface and then released above $50^{\circ}$ latitude to the cold polar traps where it will decay to ${ }^{210} \mathrm{~Pb}$ (half-life $\sim 22$ years). Thus, one would expect a significant enhancement in the emission of the $46.5 \mathrm{keV} \gamma$-ray line from ${ }^{210} \mathrm{~Pb}$ present in the lunar polar regions. Other prominent emission lines from ${ }^{234} \mathrm{Th}(63.3,92.4$ and $92.8 \mathrm{keV}),{ }^{226} \mathrm{Ra},{ }^{235} \mathrm{U}(186.2$ and $185.7 \mathrm{keV}),{ }^{228} \mathrm{Ac}(209.2 \mathrm{keV})$ and ${ }^{212} \mathrm{~Pb},{ }^{214} \mathrm{~Pb}$ ( 238.6 and $241.9 \mathrm{keV}$ ) will also be studied to obtain low spatial resolution $\mathrm{U}$ and $\mathrm{Th}$ map of the polar and U-Th enriched (e.g., KREEP) regions. We have estimated the lunar surface fluxes expected for various discrete $\gamma$ lines as well as the magnitudes of lunar continuum and detector background. These estimates suggest that a large field of view and long-term integration are essential to detect these low energy $\gamma$-ray lines from the Moon.

\section{Choice of detector and characterization}

The scintillation detectors used earlier for studying $\gamma$-ray emission from planetary surfaces (e.g., Metzger et al 1974) are not suitable for the energy range $(20-250 \mathrm{keV})$ of our interest. However, the advent of various solid-state pixelated arrays for detection of photons in this energy range has now made it feasible to attempt detection of low energy emissions from planetary surfaces. After considering various options, we narrowed our choice to the following two types of detectors, Cadmium-Telluride (CdTe) which has been flown in the INTEGRAL satellite and Cadmium-ZincTelluride (CZT) flown in the SWIFT experiment, which was launched in November 2004. The CZT detector is also being considered for ISRO's proposed Astronomical satellite, ASTROSAT. Table 1 presents the basic properties of these two detector types. As the CdTe detector exhibited lower energy resolution and higher leakage currents, we decided to choose the CZT detector for the HEX experiment. CZT arrays manufactured by IMARAD, Israel and eV Products, USA are currently being considered for use in the HEX experiment. The arrays will be coupled with ASIC designed by IDEAS, Norway. It is proposed to deploy CZT array having an effective area of $100 \mathrm{~cm}^{2}$ in the HEX experiment. The detector arrays will be maintained at $\leq 0^{\circ} \mathrm{C}$ by passive cooling to minimize thermal noise, and to achieve an energy resolution of better than $\sim 8 \%$ at $59.5 \mathrm{keV}$. A collimator providing a view of $40 \mathrm{~km} \times 40 \mathrm{~km}$ on the lunar surface will be incorporated to optimize the signal strength and spatial resolution. An active CsI 
Table 1. Comparison of CZT and CdTe detector characteristics.

\begin{tabular}{lll}
\hline \multicolumn{1}{c}{ Characteristics } & \multicolumn{1}{c}{$\mathrm{CdZnTe}(\mathrm{CZT})$} & $\mathrm{CdTe}$ \\
\hline Formula & $\mathrm{Cd}_{1-x} \mathrm{Zn}_{x} \mathrm{Te} ; x=0.1$ & $\mathrm{CdTe}$ \\
Band gap $(\mathrm{eV})$ & 1.6 & 1.44 \\
Density $(\mathrm{g} / \mathrm{cc})$ & 5.81 & 5.85 \\
Minimum energy for e-hole pair $(\mathrm{eV})$ & 4.6 & 4.43 \\
Energy resolution & $\leq 8 \%$ below $0^{\circ} \mathrm{C}$ & $50 \%$ poorer than CZT \\
Mobility & Low & Higher than CZT \\
Leakage current & Low & Higher than CZT \\
\hline
\end{tabular}

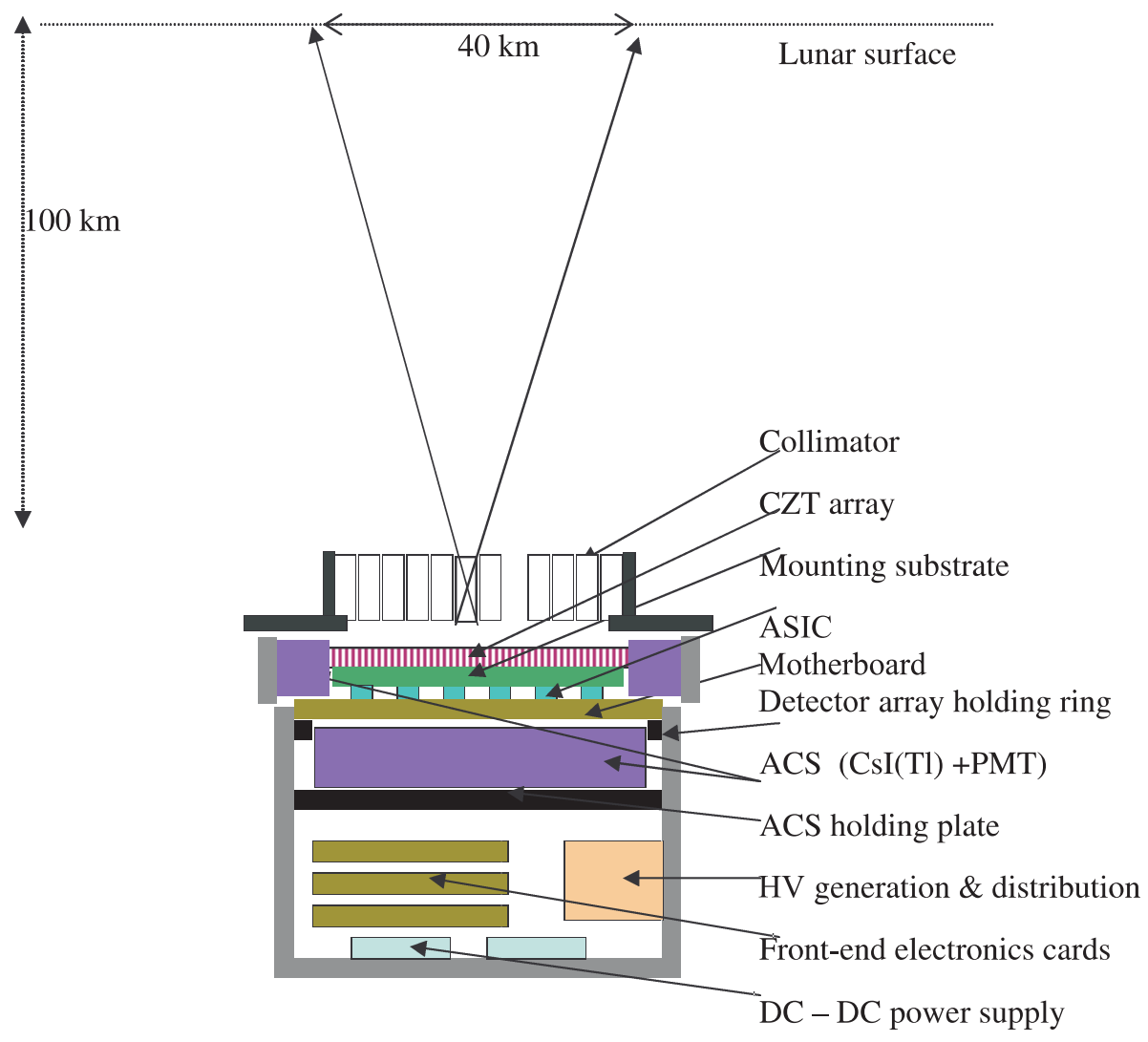

Figure 1. A schematic of the High Energy X-ray (HEX) spectrometer to be flown on Chandrayaan-1 mission. All the important sub-systems are labeled.

anti-coincidence shield will be used to minimize the detector background. Figure 1 shows an outline of the HEX spectrometer.

\section{Flux and signal calculations}

We have calculated the expected fluxes of the prominent $\gamma$ lines in the energy region $20-250 \mathrm{keV}$. The details of flux and signal calculations are given in Bhandari (2004) and Banerjee and Goswami (2004). Here, we consider the possibility of their detection using the HEX payload. To this end, we investigated the expected low energy lunar continuum background considering parameters pertinent to the HEX experiment using the CERN software GEANT 4.6.0. We consider the production of $\gamma$ rays due to interactions of energetic galactic cosmic ray protons with lunar regolith (the upper layers of lunar rocks and soils) as well as production by secondary neutrons via inelastic scattering $(n, x \gamma)$ and thermal neutron capture $(n, \gamma)$ reactions. The $\gamma$ rays escaping the lunar surface are monitored along with their kinetic energies to estimate the lunar continuum flux. We estimate the lunar continuum background to be $\sim 0.0001$ to 


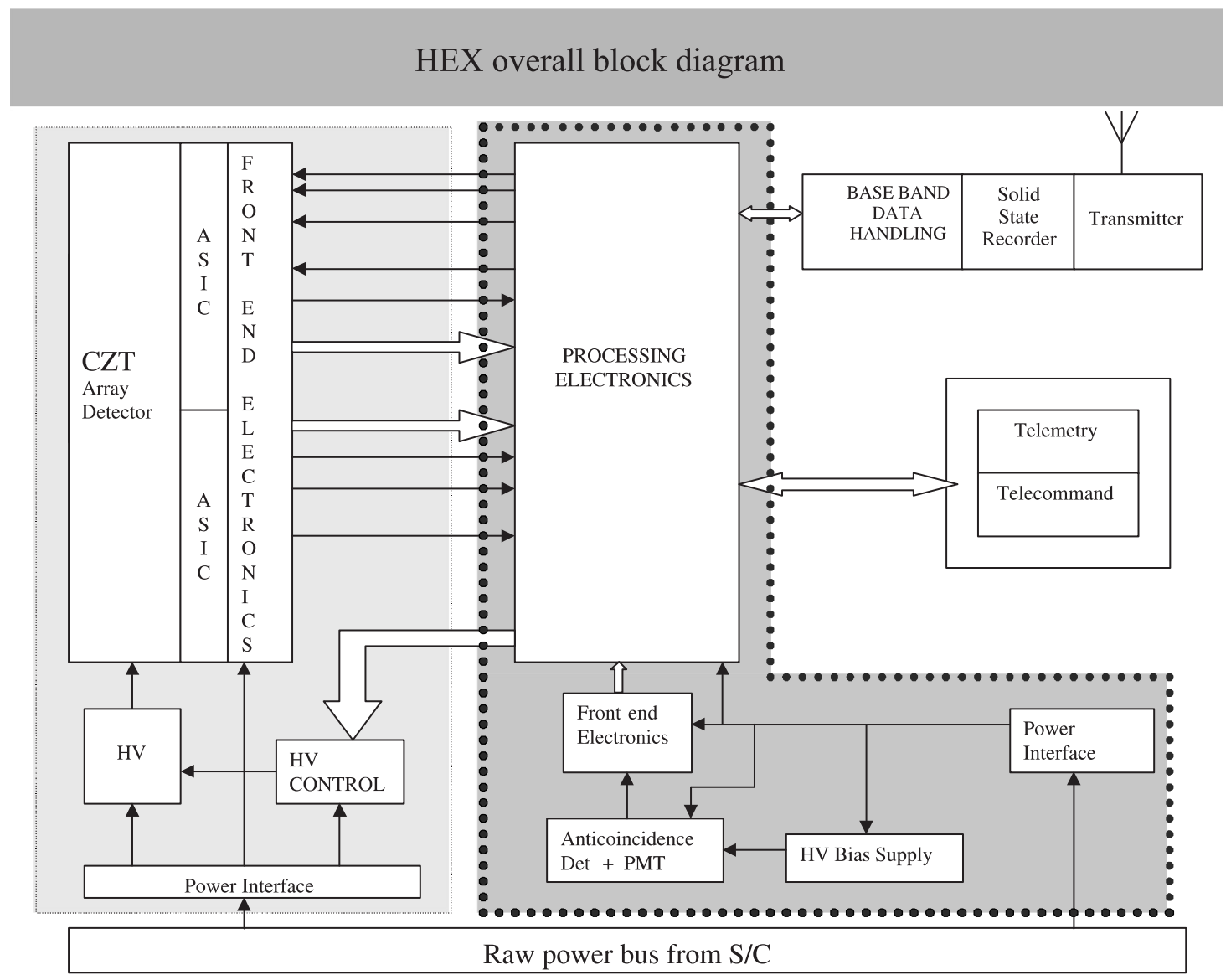

Figure 2. Overall block diagram of the HEX spectrometer.

0.006 counts $\mathrm{s}^{-1} \mathrm{~cm}^{-2} \mathrm{keV}^{-1}$ in the energy range of interest (40 to $250 \mathrm{keV}$ ) (Kim et al 2005; Banerjee 2004; unpublished data). Although no direct data are available for detector (CZT) background at this energy range and specific to space environment, background measurements with CZT detectors flown on high altitude balloon flights with active collimated shielding suggest that the detector background could be $\sim 0.0006 \mathrm{~s}^{-1} \mathrm{~cm}^{-2} \mathrm{keV}^{-1}$ in $20-40 \mathrm{keV}$ (Slavis et al 1998). Modelling of a $2 \mathrm{~mm}$ thick CZT detector surrounded by a thick BGO active shield, for a satellite in L2 orbit (1.5 million $\mathrm{km}$ from Earth) suggested that the detector background is $\sim 0.0001 \mathrm{~s}^{-1} \mathrm{~cm}^{-2} \mathrm{keV}^{-1}$ at $100 \mathrm{keV}$ (Ramsey 2001). Conservative estimates made by us indicate that integration time needed will range from 6 minutes $\left({ }^{212} \mathrm{~Pb}, 238.6 \mathrm{keV}\right)$ to 10 hours $\left({ }^{228} \mathrm{Ac}, 209.2 \mathrm{keV}\right)$ for detection of signals at $3 \sigma$ level above background if we consider emissions from the $\mathrm{U}, \mathrm{Th}$ enriched (KREEP) regions of the Moon. Nominal integration time for the $46.5 \mathrm{keV}$ line from ${ }^{210} \mathrm{~Pb}$ produced in situ in the soil will be several hours. For other terrains (highlands and mare) of the Moon, the integration times will be even higher. However, if volatile transport to colder polar region is operating on the Moon, the signal of the prime line of interest from ${ }^{210} \mathrm{~Pb}$ in the polar region is expected to be significantly enhanced by more than an order of magnitude, and the integration time will be correspondingly lower. The polar orbiting Chandrayaan-1 will facilitate integration of signal over the polar region for long durations and it should be possible to detect the enhanced signal of ${ }^{210} \mathrm{~Pb}$ and quantify the magnitude of volatile transport on the lunar surface, the prime objective of the HEX experiment. We also hope to map U, Th concentrations for the U, Th-rich KREEP region as well as in the high latitude polar region with reasonable spatial resolution. In addition, it may be possible to use the observed lunar continuum for inferring the composition of various lunar terrains (Gasnault 2004; personal communication).

\section{The experiment configuration}

The experimental configuration of the HEX spectrometer is shown in figure 1 . The effective CZT detector area of $>100 \mathrm{~cm}^{2}$ is realized by cascading nine array detectors, each $4 \mathrm{~cm} \times 4 \mathrm{~cm}$ composed of $256(16 \times 16)$ pixels 
(size: $2.5 \mathrm{~mm} \times 2.5 \mathrm{~mm} \times 3 \mathrm{~mm}$ ). Each array will be bonded to two 128 channel XAIM ASICs to be procured from IDEAS, Norway. The CsI (Tl) scintillator crystal coupled to photomultiplier tubes (PMTs) will be used as the anticoincidence detector system (ACS). It is less hygroscopic and also less fragile compared to other scintillators. The ACS is required to reduce the detector background through removal of the partially-absorbed Compton scattered events from the fully-absorbed X-ray events. The ACS is currently configured as a single piece system $(14 \mathrm{~cm} \times 14 \mathrm{~cm} \times 1 \mathrm{~cm})$ placed just below the CZT array. The detector is viewed by a pair of $2.5 \mathrm{~cm}$ PMTs mounted sideways. Using prisms, the side mounted PMTs are expected to detect the light produced in the crystal as a result of photoelectric interaction of the high energy photons with the crystal material. A tantalum-based field-of-view collimator will be mounted above the CZT arrays to limit the FOV to a $40 \mathrm{~km} \times 40 \mathrm{~km}$ swath on the lunar surface from the $100 \mathrm{~km}$ orbit of Chandrayaan-1.

\section{Electronics and electrical configurations: Basic design concept}

The block diagram of the HEX payload is shown in figure 2. The ASIC consists of charge sensitive amplifiers, shapers, and associated control circuits. The ASIC is a low noise, low power multi-channel front end integrated circuit, which is self triggered and data driven. All the output signals are of current output type. The charge collected in the CZT detectors is processed by the ASIC front end electronics (FEE) for pixel position and energy information of the detected event. These data are sent to processing electronics for further processing with telemetry. The processing electronics has its interfaces with Telemetry (TM) for monitoring various health parameters and Telecommand (TC) systems. The processing electronics design is built around FPGA namely Actel's RT54SX32SU. It is a compact package of 3 PCBs. Its main functions are programming the ASIC for CZT array, processing the data from CZT front end and ACS system. Programming the ASIC involves setting the DAC bits for various biases of the ASIC noted earlier. It includes the trimming of the data output pulse width and trigger width. Processing the data involves measuring the count rate, measuring the energy information and the time of the event. The processing electronics on command collects the pixel position, energy (ADC data), Event Tagged Time (ETT) and formats the, data into 2048 byte packets and stores in memory. The HEX experiment requires a continuous power of $\sim 25$ watts from the spacecraft power bus.

\section{Data handling}

The average count rate expected on lunar surface is quite low and will be less than 100 counts s $^{-1}$ (random) at the detector area. For every event detected, the ASIC will send out the trigger, energy and position information of the hit pixel.

\begin{tabular}{lr}
\hline Pixel position & 7 bits \\
Energy information (ADC o/p) & 8 bits \\
\# Time recorded & 12 bits \\
CZT sub-array address & 5 bits \\
Total event data & 32 bits \\
\hline
\end{tabular}

HEX data will be arranged in packets of 2048 bytes and these packets of data will be transferred to the onboard solid-state-recorder (SSR) choosing a suitable data rate. HEX incorporates two banks of memory, when one is acquiring the data, the other filled memory bank will transfer the data to FIFO and generate request flag and wait for SSR to readout the data from FIFO by sending a burst clock (2048). The transfer will be done at a rate so that by the time one memory bank is filled to $50 \%$ at the peak event rate, the other bank of memory where the data was previously acquired is fully read out by the SSR.

\section{Summary and present status}

A high energy X-ray spectrometer using new generation solid state array detector has been designed for detection of low energy $(20-250 \mathrm{keV})$ natural emission from a planetary surface for the first time. The instrument will be flown on the Chandrayaan-1 mission, scheduled for launch in late 2007, for measuring low energy $\gamma$-ray emission from the decay of $\mathrm{U}$ and $\mathrm{Th}$ on the lunar surface. A prime objective of the instrument is to study the transport of volatiles on the Moon from sunlit hotter regions to the permanently shadowed cold regions near the lunar poles by the detection of the $46.5 \mathrm{keV} \gamma$-ray line from radioactive ${ }^{210} \mathrm{~Pb}$, a decay product of the volatile ${ }^{222} \mathrm{Rn}$, both belonging to the ${ }^{238} \mathrm{U}$ series. Attempts will also be made to infer the U, Th concentrations of the polar region and U-Th-rich areas on the Moon with low spatial resolution. The possibility of inferring composition of various lunar terrains from an analysis of the lunar continuum background will also be attempted. The basic design of the payload is now complete. Breadboard versions of the various electrical and electronics modules are being made and tested. CZT arrays made by a couple of manufacturers are currently being characterized for their response function. The present plans envisage delivery of a flight model payload by early 2007 . 


\section{References}

Arnold J R, Metzger A E and Reedy R C 1977 Computergenerated maps of lunar composition from gamma-ray data; Proc. 8th Lunar Sci. Conf., pp. 945-948.

Arnold J R 1979 Ice in the lunar polar regions; J. Geophys. Res. 84 5659-5668.

Banerjee D and Goswami J N 2004 Expected signals from 20-250 keV gamma rays at $100 \mathrm{~km}$ altitude for mapping of ${ }^{210} \mathrm{~Pb}$, thorium and uranium on the lunar surface; Intern. Lunar Conf. (Abstract), Udaipur, p. 45.

Bhandari N 2004 Scientific goals of Chandrayaan-1: The Indian Lunar Polar Orbiter Mission; Curr. Sci. 86 1489-1496.

Bhandari N, Adimurthy V, Banerjee D, Srivastava N and Dhingra D 2003 Chandrayaan-1 Lunar polar orbiter: Science Goals and Payloads (ed.) Steve M Durst et al, Proc. Internat. Lunar Conference 2003, Hawaii, pp. 33-42.

Boynton W V and 24 others 2002 Distribution of Hydrogen in the Near Surface of Mars: Evidence for Subsurface Ice Deposits; Science 297 81-85.

Feldman W and 5 others 1998 Fluxes of Fast and Epithermal Neutrons from Lunar Prospector: Evidence for Water Ice at the Lunar Poles; Science 2811496.
Haines E L, Arnold J R and Metzger A E 1976 Chemical Mapping of Planetary Surfaces; IEEE Transactions of Geoscience Electronics GE-14, 3 141-153.

Kim K J, Reedy R C and Gasnault O 2005 Calculations of expected fluxes of 10-250 keV lunar leakage gamma rays; 36th Lunar Planet. Sci. Conf., Abstract no. 1900.

Lawrence D J and 6 others 1998 Global Elemental Maps of the Moon: The Lunar Prospector Gamma-Ray Spectrometer; Science 281 1484-1489.

Metzger A E, Trombka J I, Reedy R C and Arnold J R 1974 Element concentrations from lunar orbital gammaray measurements; Procs. Fifth Lunar Conf. 2 1067-1078.

Nozette S and 7 others 1996 The Clementine Bistatic Radar Experiment; Science 274 1495-1498.

Ramsey B D 2001 Fine-Pixel Imaging CdZnTe Arrays for Space Applications; Nuclear Science Symposium Conference Record, 2001 IEEE 4 2377-2381.

Slavis K R and 9 others 1998 High altitude balloon flight of CdZnTe detectors for high energy X-ray astronomy; SPIE Procs. 3445 169-183.

Stacey N J S, Campbell D B and Ford P G 1997 Arecibo radar mapping of the lunar poles: A search for ice deposits; Science 276 1527-1530.

Watson K, Murray B C and Brown H 1961 The behavior of volatiles on the lunar surface; J. Geophys. Rev. 66 3033-3045. 